\title{
Author Index, Volume 90
}

Acland GM 201

Ajmone-Marsan P 350

Albertini E 169

Allen JA 236

Altman RB 345

Alvarez G 432

Anderson AR 195

Andersone Z 17

Aransay AM 316

Arathi HS 77

Archibald JM 2

Arnaud J-F 451

Arrieta I 206

Austerlitz F 282

Bachmann K 326

Barcaccia G 169

Barnes SR 39

Barwegen MW 10

Beaulieu J 247

Beiles A 150

Benzal J 302

Beslay D 107

Bethenod M-T 141

Beukeboom LW 260

Boivin T 107

Boll R 260

Bonnavion P 157

Bourguet D 141

Bouvier JC 107

Bowman JG 39

Brakefield PM 468

Brand C 118, 204, 346

Breeuwer JAJ 130

Breyne P 268

Broadfoot LB 33

Brown RP 302

Bruford M 413

Bruins E 130

Bugrov A 377

Bunn SE 64

Burns MJ 39

Burton D 350

Cabrero J 377

Cai L 71

Camacho JPM 377

Carleton KL 116

Carton Y 84

Casulli A 17

Cavers S 268

Chadœuf J 107

Changizi M 278

Charlat S 49, 157

Charlesworth D 422

Chen X 71

Chen Z 212

Chiavarino AM 122

Chung MG 98

Chung MY 98
Ciolfi S 382

Clarke B 279

Clarke MHE 39

Clark-Tapia R 443

Cleary C 64

Cloutier D 247

Collinge JE 195

Colpaert N 268

Criado B 206

Dallai R 382

Dedryver C-A 397

de Filippis T 382

Della Rosa V 253

Delmotte F 397

Den Nijs JCM 326

de Ruijter C 10

Deunff J 451

de Vos H 468

Dijkstra C 359

di Pietro D 390

Driessen G 260

Dunner S 350

Dupas S 84

Dyson P 281

Eguiarte LE 220

Ellegren H 17

Emery SN 181

Epperson BK 98

Erickson DL 25

Etterson JR 308

Fabbri AA 253

Fahima T 150

Fajardo S 302

Falque M 326

Fanelli C 253

Flagstad Ø 17

Flores P 206

Frati F 382

Fullerton SM 121

Galloway LF 308

Garnier-Géré PH 282

Gibson G 1

Goddard ME 277

Godt MJW 187

González-Chauvet R 220

González-González E 122

González-Sánchez M 122

Gordon IJ 236

Goulson D 236

Groot TVM 130

$\mathrm{Gu} \mathrm{H} 212$

Guiller A 451

Hacia JG 343

Hackett CA 33

Halas G 390
Halkett F 397

Halverson J 17

Hamrick JL 25, 187, 308

Hanekamp E 10

Hanotte O 10

He K 212

He X 228

Hey J 343

Hikida T 90

Ho M-W 6

Hoffmann AA 195

Hu X-S 162

Hughes AL 115

Hughes JM 64

Hurwood DA 64

Irwin AJ 187

Johnson B 203

Jourde B 268

Kang L 71

Kearsey MJ 39

Keeling PJ 2

Kellett M 195

Kelly JK 77

Kirzhner VM 150

Kocher TD 116

Kojola I 17

Korol AB 150

Lapchin L 260

Le Chat L 49

Le Gallic J-F 397

Lendvai G 336

Lenstra JA 10

Levin DA 336

Li B 162, 405

Li C 71

Li D 291

Li Y-C 150

Liao $\mathrm{S} 71$

López JL 432

López-León M D 377

Lostao CM 206

Lowe AJ 268

Lucchin M 169

Luo C 405

Luo L 459

Lushai G 236

Ma X 71

Mable BK 422

Maclean N 236

Madec L 451

Marchini D 382

Martel C 141

McCauley DE 181

McKechnie SW 195

Meats A 365 
Melis V 298

Meneghetti S 169

Merçot H 49, 157

Mes THM 326

Mezzanotte R 298

Molina F 122

Molina-Freaner F 220, 443

Mommens G 350

Morillas-Marquez F 316

Mosquera E 432

Müller C 468

Nason JD 98

Navarro C 268

Navarro-Quezada A 220

Nei M 411

Nevo E 150

Nieddu M 298

Nijman IJ 10, 350

Ochieng JW 10

O'Connor CD 415

Okada S 90

Ortega B 206

Ostrander EA 201

Ota H 90

Otsen M 10

Oxford G 348

Palladino MA 9

Papura D 397

Peixoto A L 206

Peñagarikano O 206

Perfectti F 377

Pestano J 302

Pichiri G 298

Pike N 365

Poiriè M 84

Porter DM 416

Pozzi C 390

Puertas MJ 122
Qu L-J 212

Quilici S 382

Radwan J 371

Ready PD 316

Rege JEO 10

Réjasse A 141

Reverberi M 253

Richards CM 181

Rioux D 247

Roberts H 291

Rodellar C 350

Röder MS 150

Roig C 390

Rosato M 122

Rosetto M 382

Roulin A 359

Rousset F 141

Salamini F 390

Sasakuma T 56

Sauphanor B 107

Schierup MH 422

Schilling T 4

Schmidt O 291

Schneider MV 260, 291

Schoen DJ 247

Selzner A 260

Shamshad S 10

Shen D 71

Shindo C 56

Sifferd KL 418

Simon J-C 397

Smith DAS 236

Smith KK 8

Smouse PE 187

Somerville L 421

Sonti RV 120

Stam P 326

Su H 212

Suárez NM 302
Sulawati T 10

Sun L 71

Sundqvist A-K 17

Talsma J 260

Télez M 206

Theopold U 291

Toda M 90

Triest L 169

Tsujimoto H 56

Urbanelli S 253

Valdmann H 17

Valentini A 350

Van Der Hulst RGM 326

van Eunen K 260

Veiga I 206

Verkaar ELC 10

Vilà C 17

Walker C 17

Wallace BMN 136

Wallace H 136

Wang J 212

Wang WYS 365

Warchalowska-Śliwa E 377

Wenstrom K 419

Werner CP 39

Wiener P 350

Williams JL 350

Wilson J 268

Xu C 228

Xu S 228, 459

Zhang Z 212

Zhao S 71

Zhao Z 291

Zhu H 71

Zwaan BJ 468 